\title{
Crises des solidarités ou solidarités dans la crise ? Une lecture à partir de l'analyse de l'évolution de la structure des ménages entre 1993 et 2003 à Ouagadougou, Burkina Faso.
}

\author{
Par \\ Banza Baya \\ Maître de conférences, Université de Ouagadougou \\ (ISSP), Burkina Faso
}

\section{Résumé}

Cet article appréhende le système de solidarité dans un contexte de crise urbaine, à travers l'analyse de l'évolution de la structure et de la taille des ménages à Ouagadougou à partir des Enquêtes démographiques et de santé (EDS) réalisées au Burkina Faso (1993, 1998/L999 et 2003). Il teste les hypothèses de nucléarisation et de diminution de la taille des ménages, et identifie les déterminants des ménages nucléaires et de faible taille au moyen de deux régressions logistiques. Les résultats indiquent que bien que les ménages nucléaires augmentent légèrement, la majorité des ménages demeurentélargis. Bien plus, la tendance à la nucléarisation est plus faible chez les ménages mieux pourvus en ressources (que ce soit en termes d'éducation ou de niveau de vie). Ceci suggère une continuation des solidarités en période de crise.

\begin{abstract}
This study investigates solidarity systems in a context of urban crisis, through an analysis of trends in household's structure and size in Ouagadougou across three DHS surveys (1993, 1998A999 and 2003). It tests the hypotheses of nuclearization and household size reduction, and identifies the determinants of household size and extension by using two logistic regression models. Results indicate that most households remain large in Ouagadougou, despite a slight increase in the percent of nuclear households during the period. More importantly, the better endowed households (in terms of household head's education and household standard of living) are more likely to remain extended. Such results suggest a continued solidarity during periods of economic downturn.
\end{abstract}




\section{Introduction}

Unité sociale de base de toute société, la famille constitue à la fois le cadre de procréation, de socialisation, et d'éducation des enfants (Nations unies 1995; Develay et al. 1996; Debpuur 1998; Bongaarts 2001). A ce titre, elle est déterminante dans la reproduction et le changement démographique en Afrique. De sa structure, dépend l'évolution de la transition africaine amorcée depuis les années 1990. Historiquement, ce système de famille étendue est supposé évoluer sous l'influence de l'urbanisation et la modernisation. La théorie de la nucléarisation (Pilon et Vignikin 2006) suppose en effet une convergence des systèmes familiaux vers le modèle nucléaire en conséquence de la modernisation des structures économiques et sociales.

La confrontation du modèle de convergence des structures familiales avec des données empiriques révèle toutefois que le schéma d'évolution de la famille africaine vers la nucléarisation n'est pas toujours respecté (Cordell et Piché 1997). Le modèle de famille étendue se maintient parfois durant le processus de modernisation, alors que les ménages les plus modernes sont parfois les plus étendus. De plus en plus de recherches mettent en évidence la diversification des modes de structurations familiaux et l'existence de disparités selon les pays et les milieux de résidence (Pilon et Vignikin 2006). Bongaarts (2001 : 266) a quant à lui relevé dans les pays en développement des tailles de ménage semblables à celles qui furent observées en Europe et en Amérique du Nord durant la seconde moitié du 19è siècle.

Bien que la législation de la famille favorise la famille nucléaire dans les pays de l'Afrique sub-saharienne francophone post coloniale, les ménages ouest-africains sont plus étendus (Adjamagbo-Johnson 1997). En raison de l'importance revêtue par la famille pour le bien-être de ses membres, il convient de se demander si la dégradation de la situation économique des dernières décennies a conduit à un repli sur la famille conjugale ou au renforcement du système traditionnel de solidarité. En effet, certaines stratégies familiales vont à l'encontre de la nucléarisation. La famille continuerait, dans certains contextes subsahariens, de jouer son rôle de refuge et d'assurance sociale, comme au Burkina Faso. Même si quelques travaux ont examiné les changements dans le système de famille étendue dans un contexte de crise, l'accent a surtout été mis sur les échanges de ressources entre familles ou le confiage d'enfants. Cette étude complète les travaux antérieurs en examinant l'évolution de la composition des ménages.

L'examen de cette évolution permet de vérifier l'hypothèse de nucléarisation des familles. C'est ainsi que notre étude vérifie la pertinence des thèses de repli vers le mode conjugal ou de renforcement des solidarités familiales au Burkina Faso, en déterminant si un processus de nucléarisation des ménages a eu lieu à Ouagadougou de 1993 à 2003. 
Banza Baya, Danièle Laliberté: Crises des solidarités ou solidarités dans la crise ? Une lecture à partir de l'analyse de l'évolution de la structure des ménages entre 1993 et 2003 à Ouagadougou, Burkina Faso.

\section{Revue de la littérature et cadre théorique}

Structures familiales, familles et ménages

La famille est un objet d'étude privilégié en sciences sociales, en raison de son rôle fondamental dans le processus de socialisation. Les études empiriques ont révélé la diversification et la complexité des systèmes familiaux à travers le temps et l'espace. Plus facile à cerner empiriquement, le concept de ménage est devenu un proxy de la famille dans les recherches démographiques. Les critères de définition du ménage font généralement référence aux dimensions de résidence, de filiation et d'alliance, de communauté de vie, de procréation, de production et de consommation.

Cependant, le ménage n'est qu'une approximation du concept de famille, plus particulièrement en contexte africain où la famille étendue intervient dans la socialisation, la production et la reproduction sociales. Qui plus est, le critère de co-résidence, retenu dans la définition usuelle du ménage, est contestable lorsque les unités de procréation, de production et de consommation opèrent dans un espace multi-résidentiel, ce qui est fréquent dans un contexte polygamique (Roy 1985:6-7).

Cadres théoriques et tendances observées

La convergence des structures familiales vers un modèle unique, celui de la famille nucléaire, a été anticipée sur la base des faits observés en Occident aux dix- huitième et dix-neuvième siècles. Cependant, la conceptualisation $\mathrm{du}$ processus de nucléarisation varie d'un courant théorique à l'autre. La théorie de la modernisation oppose la famille nucléaire néolocale à la famille étendue de la société traditionnelle, composée de plusieurs familles nucléaires ou de familles polygamiques partageant un lieu de résidence.

\section{Selon 1'approche structuro-} fonctionnaliste, la nucléarisation est une réponse aux besoins de la société industrielle et résulte de la transformation des structures économiques et sociales (Parsons et Baies 1955). Pour l'approche culturaliste et diffusionniste, centrée sur l'individu et le couple, les facteurs culturels déterminent les changements de structure familiale. Les valeurs sont modernisées par la diffusion des idées et technologies de l'Occident vers les pays pauvres, puis de l'élite urbaine à l'élite rurale. L'urbanisation sert en quelque sorte de véhicule transformant les comportements traditionnels en comportements " modernes».

La théorie du développement rural (Kocher 1973) attribue quant à elle la nucléarisation à l'amélioration des conditions de vie et des ressources (revenus, santé et éducation) associés à la modernisation. Selon la théorie des flux intergénérationnels de richesse (Caldwell 1976 et 1978), la taille de la famille est d'abord déterminée par des facteurs personnels, sociaux et psychologiques. La nucléarisation surviendrait après l'adoption de nouvelles valeurs véhiculées 
par l'école et les mass média. La révolution sociale des relations familiales et plus particulièrement la direction des flux de richesse intrafamiliaux dictés par les obligations familiales survient en général simultanément ou préalablement à la modernisation économique (Caldwell 1976 :322).

Enfin, l'approche historicostructurelle relie la transformation de la famille aux stratégies de reproduction élaborées pour accomplir les activités de production et de reproduction (Poirier 1986). L'introduction de rapports de production capitalistes transforme les conditions de la reproduction, les structures familiales et les relations au sein des familles.

L'évolution des ménages dépend donc de facteurs sociaux, culturels, économiques et politiques. Elle est reliée aux régimes de fécondité et de mortalité, aux régimes matrimoniaux et migratoires, aux modes de production, aux pratiques résidentielles et à l'habitat, aux normes familiales et aux règles d'héritage, au sexe et à l'âge du chef de ménage.

L'importance relative des ménages de cinq personnes et plus dans le monde a diminué entre 1970 et 1980 (Nations unies 1995). L'analyse des EDS de quarante-trois pays en développement (dont le Burkina Faso) entre 1990 et 1998 par Bongaarts (2001) indique une nucléarisation des ménages et une réduction de leur taille. Par contre, des études longitudinales ont récemment dévoilé la présence de ménages de grande taille à structure complexe, dans les pays du Sud et du Nord (Peek et al. 2004).

Si la convergence vers un système familial nucléaire avait lieu, la taille et la complexité des ménages seraient moindres en milieu urbain et dans les couches de population les plus instruites (Bongaarts 2001: 274). Cependant, les écarts entre zones urbaines et rurales et selon l'éducation sont minimes. Par ailleurs, Pilon et Vignikin (2006) anticipent que l'amélioration de la scolarisation sur le continent africain conduira inéluctablement à la nucléarisation des familles et à l'instauration d'un univers familial de plus en plus privé et calqué sur le modèle occidental.

Par ailleurs, les problèmes économiques vécus dans les sociétés africaines amènent des adultes à s'associer dans le même espace résidentiel pour assurer la subsistance du groupe (Locoh 1988). Ces solidarités familiales, qui contribuent au maintien des familles étendues en milieu urbain, visent à affronter les difficultés financières dues à l'instabilité et la faiblesse des revenus, au coût élevé des loyers et aux problèmes d'accès à la propriété, au crédit et à l'emploi. Des ménages hébergent des migrants en vue de faciliter leur insertion, reçoivent des enfants confiés pour leur scolarité ou comme main-d'œuvre domestique et intègrent des femmes en rupture d'union. 
Banza Baya, Danièle Laliberté: Crises des solidarités ou solidarités dans la crise ? Une lecture à partir de l'analyse de l'évolution de la structure des ménages entre 1993 et 2003 à Ouagadougou, Burkina Faso.

L'évolution des structures familiales au Burkina-Faso

Au milieu des années soixante-dix (1974-1975), les ménages voltaïques uniquement composés de la famille du chef ne constituaient que $46,5 \%$ des ménages ayant fait l'objet de l'Enquête migratoire Haute-Volta -- actuel Burkina-Faso (Roy 1985). On remarquait alors l'absence d'un schéma d'évolution général et d'une définition unique du ménage, l'existence de structures familiales complexes et diversifiées, la présence de familles nombreuses, la fréquente pratique de la polygamie et la cohabitation de plusieurs générations.

À partir des données d'une enquête réalisée en 2000 à Bobo-Dioulasso, seconde ville du Burkina Faso, Baya (2003) a observé que la taille moyenne des ménages est de 5,6 comparativement à 7,0 en milieu rural. Alors que les ménages de neuf personnes et plus représentent près du tiers de l'ensemble des ménages en milieu rural, ils ne comptent que pour $19 \%$ des ménages en milieu urbain.

L'incidence de la pauvreté en milieu urbain au Burkina Faso a presque doublé entre 1994 et 2003 (Burkina Faso 2003a et Burkina Faso 2003b). L'économie nationale a stagné pendant deux décennies, compte tenu de sa faible capacité à répondre aux chocs extérieurs en raison même de contraintes et de limites intrinsèques telles que la dévaluation et les conditions climatiques défavorables. Les difficultés économiques ont suscité la création de nouveaux modes de logement faisant appel aux relations de parenté ou à des liens hors parenté. Elles ont engendré de nouvelles formes de concessions où la parentèle n'est plus l'unique élément de structuration.

La présente étude vise l'analyse de l'évolution de la taille et de la composition des ménages de Ouagadougou. Elle vérifie les hypothèses de nucléarisation de la famille et de réduction de sa taille entre 1993 et 2003, et détermine l'effet des principaux facteurs explicatifs dégagés de la revue de la littérature. En examinant la propension de la population ouagalaise à former des ménages nucléaires, ou au contraire à inclure des membres dont les liens de parenté sont inexistants ou distants du noyau familial, on confronte les thèses du repli sur la famille conjugale et de la manifestation d'un renforcement des solidarités familiales.

\section{Données et méthodes}

Les questions de recherche sont explorées à partir des trois EDS réalisées au Burkina Faso en 1993 (5143 ménages dont 1 242 à Ouagadougou), 1998/1999 (4 812 ménages dont 745 à Ouagadougou) et 2003 (9097 ménages dont 478 à Ouagadougou). Préalablement à l'administration du questionnaire individuel destiné aux femmes éligibles du ménage (15-49 ans) et à quelques-uns des époux (15-59 ans), un questionnaire ménage a été administré à tous les ménages sélectionnés. Le ménage à Ouagadougou est l'unité d'analyse de la présente étude. Il est important de noter que les variations d'échantillonnage d'une enquête à l'autre constituent une contrainte 
African Population Studies Vol. $22 n^{\circ}$ 2/Etude de la Population Africaine Vol. $23 N^{*} 2$

méthodologique, plus particulièrement la taille nettement inférieure de l'échantillon en 2003.

Les variables dépendantes et indépendantes de cette étude ont été dichotomisées aux fins de l'analyse multivariée. La première variable dépendante est le type de ménage, et ses modalités sont "nucléaire » et " non nucléaire ». Les ménages nucléaires sont ceux constitués des ménages « biologiques / recomposés », des ménages formés d'une seule personne et des ménages monoparentaux (avec enfants mais sans conjoint(e)s). Les ménages non nucléaires comportent d'autres personnes en plus ou à la place des membres du ménage nucléaire. La deuxième variable dépendante est la taille du ménage : les ménages sont dits de petite taille lorsque cette dernière est inférieure à cinq personnes, et de grande taille si égale ou supérieure à cinq personnes. Le statut socio-économique du ménage est cerné par un proxy construit à partir des caractéristiques du logement.

La stratégie d'analyse consiste à vérifier les hypothèses de nucléarisation et de diminution de la taille des ménages, en identifiant leurs déterminants par le biais de deux régressions logistiques où les coefficients estimés sont présentés sous la forme de rapports de chance.

\section{Résultats}

Analyse descriptive

Le tableau 1 présente la répartition des ménages de Ouagadougou selon les variables retenues dans les modèles de régression, et résume les résultats des analyses descriptives. Par souci de concision, les résultats de certaines analyses descriptives sont incorporés dans le texte sans toutefois faire l'objet d'un tableau.

Structure par âge et sexe

Dans l'ensemble, l'âge moyen des chefs de ménage à Ouagadougou a augmenté de 1993 (40,1 ans) à 2003 (41,3 ans), de même que l'écart d'âge entre les deux sexes, qui est passé de 2,2 (1993) à 5,1 (1998/1999) puis à 4,1 (2003). En moyenne, les femmes chefs de ménage sont plus âgées que leurs homologues hommes ; celles de 50 ans et plus représentent $30 \%$, comparativement à moins de $25 \%$ pour les hommes. Leur âge moyen est supérieur à celui des hommes chefs, signe qu'elles deviennent souvent chefs en l'absence de leur conjoint. Plus des quatre-cinquièmes des Ouagalais(e)s vivent dans des ménages dirigés par des hommes ; toutefois, la proportion de la population dont le chef de ménage est une femme augmente de 1993 $(9,6 \%)$ à $1998(15 \%)$ puis à $2003(16,1 \%)$. Plus de $80 \%$ des ménages demeurent donc dirigés par un homme. Cependant, en 2003 les chefs de ménage de sexe féminin constituent près de $17 \%$ comparativement à moins de $12 \%$ en 1993 .

\section{Taille du ménage}

La taille moyenne des ménages a diminué historiquement en milieu urbain (de 6,1 à 5,6), y compris à Ouagadougou $(5,9$ à 5,5$)$, où la taille moyenne des 
Banza Baya, Danièle Laliberté: Crises des solidarités ou solidarités dans la crise ? Une lecture à partir de l'analyse de l'évolution de la structure des ménages entre 1993 et 2003 à Ouagadougou, Burkina Faso.

ménages est relativement plus faible. La proportion de ménages de grande taille (dix personnes et plus) est passée d'environ $16,4 \%$ en 1993 à près de $12,6 \%$ en 1998 et 2003. Par contre, les ménages de moins de cinq personnes ont augmenté: d'un peu plus de $42 \%$ en 1993 , ils atteignent près de $47 \%$ en 2003. On observe deux tendances selon le sexe du chef ; contrairement aux ménages dirigés par des hommes, la taille de ceux des femmes augmente $(4,8$ à 5,2 personnes). Toutefois, dans l'ensemble, la taille moyenne baisse légèrement, en raison de l'importance numérique des ménages dirigés par des hommes.

\section{Type de ménage}

Conformément à la théorie de la nucléarisation, la proportion des ménages nucléaires augmente à Ouagadougou, quoique faiblement, de 40,5\% (1993) à $41,9 \%$ (1998/1999) et à 43,4\% (2003). Elle est nettement plus élevée chez les chefs de ménage de sexe masculin $(41,5 \%$ contre $33,1 \%$ en $1993 ; 45,0 \%$ contre $26,6 \%$ en $1998 / 1999$ et $46,9 \%$ contre $27,2 \%$ en 2003 ). Les autres ménages renferment près d'un tiers ou moins de membres de la famille nucléaire. En d'autres termes, la complexité de la structure des ménages des femmes est plus grande et s'accentue dans le temps. Ainsi, les " autres parents » y sont davantage représentés, la proportion de ménages en comportant, étant de 17\% (1993), 16\% (1998/1999) et 18\% (2003), comparativement à seulement $12 \%$ pour les hommes.

\section{Instruction et niveau socio-économique}

lors que les chefs de ménage sont de plus en plus instruits au fil du temps, l'écart entre les chefs de sexe masculin et féminin se réduit considérablement. De plus, la proportion des ménages de moins de cinq personnes est nettement plus élevée chez les chefs de ménages de niveau secondaire et au-delà $(55,4 \%)$. Parallèlement, la proportion des ménages de moins de cinq personnes est minoritaire pour les chefs sans instruction ou de niveau primaire. Les ménages comportant dix membres et plus augmentent chez les chefs d'instruction primaire de 1993 (12,9\%) à 2003(14,3\%).

Les ménages nantis comptent majoritairement cinq à neuf personnes (près de 50\%). Par contre, les ménages pauvres ont une taille inférieure à cinq, à raison de 44\% (1993), de 47,4\% (1998/1999) et de $46,6 \%$ (2003). Enfin, les ménages intermédiaires se répartissent presqu'également entre la taille inférieure à cinq et la taille allant de cinq à neuf en 1993, pour ensuite se retrouver essentiellement dans les ménages de petite taille en $19984999(46,5 \%$ ) et en $2003(46,8 \%$ ).

La nucléarisation est plus fréquente chez les chefs sans instruction $(44,8 \%$ en 1993 et $48,1 \%$ en 19984999 ), sauf en 2003 , où la proportion de ménages nucléaires la plus élevée est pour l'instruction primaire $(51,1 \%)$. La proportion des ménages nucléaires la plus faible est chez les chefs de niveau secondaire et plus, où elle ne dépasse pas $35,5 \%$. Enfin, la propension à diriger un ménage composé à la fois de 
dépasse pas 35,5\%. Enfin, la propension à diriger un ménage composé à la fois de parents et de non parents est toujours plus élevée pour l'instruction secondaire et plus.

Tableau 1 : Répartition (en \%) des ménages de Ouagadougou selon les variables dépendantes et indépendantes en 1993, 1998/1999 et 2003

\begin{tabular}{|c|c|c|c|}
\hline \multicolumn{4}{|c|}{ VARIABLE DÉPENDANTE } \\
\hline & EDS 1993 & EDS 1998/1999 & EDS 2003 \\
\hline $\begin{array}{l}\text { Type de ménage } \\
\text { Nucléaire } \\
\text { Non nucléaire }\end{array}$ & $\begin{array}{l}40,5 \\
59,5\end{array}$ & $\begin{array}{l}41,9 \\
58,1\end{array}$ & $\begin{array}{l}43,3 \\
56,7\end{array}$ \\
\hline $\begin{array}{l}\text { Taille du ménage } \\
\text { Moyenne } \\
\text { Moins de } 5 \text { personnes } \\
5 \text { personnes et }+\end{array}$ & $\begin{array}{l}5,9 \text { personnes } \\
42,0 \\
58,0\end{array}$ & $\begin{array}{l}5,6 \text { personnes } \\
45,8 \\
54,2\end{array}$ & $\begin{array}{l}5,5 \text { personnes } \\
46,8 \\
53,2\end{array}$ \\
\hline \multicolumn{4}{|c|}{ VARIABLES INDÉPENDANTES } \\
\hline & EDS 1993 & EDS 1998/1999 & EDS 2003 \\
\hline \multicolumn{4}{|c|}{ Caractéristiques du ménage } \\
\hline $\begin{array}{l}\text { Niveau socio- } \\
\text { économique } \\
\text { Pauvre } \\
\text { Intermédiaire } \\
\text { Nantis }\end{array}$ & $\begin{array}{r}43,6 \\
49,4 \\
7,0\end{array}$ & $\begin{array}{l}42,1 \\
46,2 \\
11,7\end{array}$ & $\begin{array}{c}40,5 \\
59,5 \\
0\end{array}$ \\
\hline \multicolumn{4}{|c|}{ Caractéristiques du chef de ménage } \\
\hline $\begin{array}{l}\text { Sexe } \\
\text { Femme } \\
\text { Homme }\end{array}$ & $\begin{array}{l}11,7 \\
88,3\end{array}$ & $\begin{array}{r}16,6 \\
83,4 \\
\end{array}$ & $\begin{array}{l}16,9 \\
83,1\end{array}$ \\
\hline $\begin{array}{l}\text { Age } \\
15-29 \text { ans } \\
30-39 \text { ans } \\
40-49 \text { ans } \\
50-59 \text { ans } \\
60 \text { ans et }+\end{array}$ & $\begin{array}{r}19,9 \\
34,9 \\
22,8 \\
14,7 \\
7,7\end{array}$ & $\begin{array}{r}23,3 \\
31,1 \\
26,2 \\
12,1 \\
8,3\end{array}$ & $\begin{array}{l}17,4 \\
32,9 \\
24,9 \\
13,8 \\
10,9\end{array}$ \\
\hline $\begin{array}{l}\text { Niveau d'instruction } \\
\text { Sans instruction } \\
\text { Primaire } \\
\text { Secondaire et + }\end{array}$ & $\begin{array}{l}52,9 \\
17,5 \\
29,6\end{array}$ & $\begin{array}{l}46,8 \\
18,3 \\
34,9\end{array}$ & $\begin{array}{l}38,0 \\
19,1 \\
42,9\end{array}$ \\
\hline $\begin{array}{l}\text { Statut matrimonial } \\
\text { Jamais marié(e) } \\
\text { Divorcé(e), séparé(e) } \\
\text { ou veuf(ve) } \\
\text { Actuellement marié(e) }\end{array}$ & $\begin{array}{r}8,8 \\
15,2 \\
76,0\end{array}$ & $\begin{array}{c}8,8 \\
19,1 \\
72,1\end{array}$ & $\begin{array}{l}---- \\
---- \\
-----\end{array}$ \\
\hline
\end{tabular}

Sources : EDS du Burkina Faso 1993, 1998/1999 et 2003. 
Banza Baya, Danièle Laliberté: Crises des solidarités ou solidarités dans la crise ? Une lecture à partir de l'analyse de l'évolution de la structure des ménages entre 1993 et 2003 à Ouagadougou, Burkina Faso.

Analyse explicative

Facteurs de nucléarisation des ménages Le tableau 2 récapitule les effets de plusieurs variables sur le type de ménage à Ouagadougou. L'analyse confirme l'importance du sexe du chef de ménage, les ménages dirigés par un homme ayant entre 1,4 et 2,3 fois plus de chance d'être nucléaires. Les chances d'appartenir à un ménage nucléaire augmentent légèrement de 1993 à 2003. L'effet de l'âge est généralement significatif (sauf dans deux cas) ; plus le chef de ménage avance en âge, moins il est susceptible de vivre dans un ménage nucléaire, surtout en 2003. Les chefs âgés de 60 ans et plus ont $92 \%$ de chances en moins que les jeunes de 15 à 29 ans de diriger un ménage nucléaire.

La nucléarisation est moins fréquente parmi les chefs de niveau primaire (comparés à ceux sans instruction), et encore moindre pour ceux avec le niveau secondaire et plus. En 1993, un chef d'instruction primaire a $26 \%$ ou $24 \%$ moins de chance (dépendamment des modèles) qu'un chef sans instruction dediriger un ménage nucléaire, et 33\% en 19984999. Les ménages avec un chef de niveau secondaire ont nettement moins de chance d'être nucléaires, avec des rapports de chance de l'ordre de 0,50 (0,52 dans le second modèle) en 1993, comparativement à 0,42 (0,45 dans le deuxième modèle) en 1998/1999, et 0,47 en 2003. Par ailleurs, le statut matrimonial n'est pas significatif. Les coefficients relatifs a niveau socioéconomique du ménage varient d'une enquête à l'autre. Ainsi, dépendamment des modèles, comparativement aux ménages pauvres, les ménages nantis ont $44 \%$ à $46 \%$ moins de chance d'être nucléaires en 1993, comparativement à 41 $\%$ et $43 \%$ en 19984999 . Le coefficient du niveau intermédiaire n'est significatif qu'en 2003, associé à plus de deux fois plus de chances d'être dans un ménage nucléaire que pour les ménages pauvres.s. 
Tableau 2 : Rapports de chance des variables de la régression logistique sur le type de ménage, Ouagadougou, 1993, 1998/99 et 2003

\begin{tabular}{|c|c|c|c|c|c|}
\hline \multicolumn{6}{|c|}{ VARIABLE DÉPENDANTE } \\
\hline \multicolumn{6}{|l|}{ Type de ménage } \\
\hline \multicolumn{6}{|c|}{$\begin{array}{l}\text { (1) Nucléaire (chef seul ou avec présence de conjoint(e)s et/ou d'enfants } \\
\text { biologiques) } \\
\text { (0) Non nucléaire (présence d'autres personnes) }\end{array}$} \\
\hline & \multicolumn{2}{|c|}{ EDS 1993} & \multicolumn{2}{|c|}{ EDS 199899} & EDS 2003 \\
\hline \multicolumn{6}{|c|}{ VARIABLES INDÉPENDANTES } \\
\hline \multicolumn{6}{|c|}{ Caractéristiques du ménage } \\
\hline \multicolumn{6}{|l|}{$\begin{array}{l}\text { Niveau socio- } \\
\text { économique } \\
\text { (Pauvre) }\end{array}$} \\
\hline Intermédiaire & 0,93 & 0,93 & 0,93 & 0,94 & $1,92 * * *$ \\
\hline Nanti & $0,56 * *$ & $0,54 * *$ & $0,59 *$ & $0,57 *$ & --- \\
\hline \multicolumn{6}{|c|}{ Caractéristiques du chef de ménage } \\
\hline $\begin{array}{l}\text { Sexe } \\
\text { (Femme) } \\
\text { Homme }\end{array}$ & $1.82 * *$ & $1,41 *$ & $1.92 * *$ & $2.07 * * *$ & $2,32 * * *$ \\
\hline \multicolumn{6}{|l|}{$\hat{A g e}$} \\
\hline $\begin{array}{l}\text { (15 à } 29 \text { ans) } \\
30-39 \text { ans }\end{array}$ & $071 *$ & & & & \\
\hline $\begin{array}{l}30-39 \text { ans } \\
40-49 \text { ans }\end{array}$ & $0,44 * * *$ & $0,00^{* * *}+40^{* * *}$ & 0.69 & $0,59 *$ & $0,54 * *$ \\
\hline $50-59$ ans & $0,51 * * *$ & $0,47 * * *$ & $0,47 * *$ & $0,39 * * *$ & $0,32 * * *$ \\
\hline 60 ans et + & $0,32 * * *$ & $0,29 * * *$ & $0,33 * * *$ & $0,45 * * *$ & $0,08 * * *$ \\
\hline \multicolumn{6}{|l|}{ Niveau d'instruction } \\
\hline Primaire & $0,74^{*}$ & $0,76^{*}$ & $0,67 *$ & $0,67 *$ & 0,92 \\
\hline Secondaire et + & $0,50 * * *$ & $0,52 * * *$ & $0,42 * * *$ & $0,45 * * *$ & $0,47 * * *$ \\
\hline \multicolumn{6}{|l|}{ Statut matrimonial } \\
\hline $\begin{array}{l}\text { Actuellement } \\
\text { marié(e) }\end{array}$ & 0,80 & & 0,76 & & \\
\hline $\begin{array}{l}\text { Divorcé(e), séparé(e) } \\
\text { ou veuf(ve) }\end{array}$ & 1,29 & & 0,67 & & \\
\hline
\end{tabular}

Sources : EDS du Burkina Faso 1993, 1998/1999 et 2003 (Burkina Faso 1994, 2000 et 2004).

Note : Seuils de signification : * 10\% (z: $\pm 1,645), * * 5 \%(\mathrm{z}: \pm 1,96), * * * 1 \%(\mathrm{z}: \pm 2,58)$.

Acteurs influençant la taille des ménages

Les effets des variables retenues sur la taille du ménage sont présentés au tableau 3. Il ressort ici également que les ménages dirigés par des hommes ont tendance à être de grande taille comparativement à ceux ayant une femme à leur tête (les rapports de chance significatifs varient de 0,47 à 0,65 en 1993 ett 
Banza Baya, Danièle Laliberté: Crises des solidarités ou solidarités dans la crise ? Une lecture à partir de l'analyse de l'évolution de la structure des ménages entre 1993 et 2003 à Ouagadougou, Burkina Faso.

1998/1999). Comparativement aux chefs âgés de moins de 30 ans, les chefs de ménage plus âgés ont plus de chance de diriger des ménages de grande taille. En 1993 et 1998/1999, les ménages où le chef est célibataire ont, comme on pouvait s'y attendre, significativement plus de chance d'être dans un ménage de petite taille.
Les chefs de niveau primaire ont entre 33\% et $37 \%$ (dépendamment des modèles) moins de chance d'être dans des ménages de taille réduite que les sans instruction en 1993 et en 1998/1999. Enfin, en 1993 et 19984999, les ménages nantis tendent à être plus grands que les pauvres, les rapports de chance variant de 0,56 à 0,59 .

Tableau 3 : Rapports de chance des variables de la régression logistique sur la taille des ménages, Ouagadougou, 1993, 1998/99 et 2003

\begin{tabular}{|c|c|c|c|c|c|}
\hline \multicolumn{6}{|c|}{ VARIABLE DÉPENDANTE } \\
\hline \multirow{2}{*}{\multicolumn{6}{|c|}{$\begin{array}{l}\text { Taille des ménages } \\
\text { (1) Ménage de taille réduite (moins de } 5 \text { personnes) } \\
\text { (0) Ménage de grande taille ( } 5 \text { personnes et }+ \text { ) }\end{array}$}} \\
\hline & & & & & \\
\hline & \multicolumn{2}{|c|}{ EDS 1993} & \multicolumn{2}{|c|}{ EDS 199899} & EDS 2003 \\
\hline \multicolumn{6}{|c|}{ VARIABLES INDÉPENDANTES } \\
\hline \multicolumn{6}{|c|}{ Caractéristiques du ménage } \\
\hline $\begin{array}{l}\text { Niveau socio- } \\
\text { économique } \\
\text { (Pauvre) } \\
\text { Intermédiaire } \\
\text { Nantis }\end{array}$ & $\begin{array}{l}0,93 \\
0,59 \\
\end{array}$ & $\begin{array}{l}0,93 \\
0,56^{*}\end{array}$ & $\begin{array}{l}0,87 \\
0,68\end{array}$ & $\begin{array}{l}0,87 \\
0,59 *\end{array}$ & \begin{tabular}{|l|}
1,10 \\
---
\end{tabular} \\
\hline \multicolumn{6}{|c|}{ Caractéristiques du chef de ménage } \\
\hline $\begin{array}{l}\text { Sexe } \\
\text { (Femme) } \\
\text { Homme }\end{array}$ & 1,03 & $0,47 * * *$ & 0,75 & $0,65^{*}$ & 0,74 \\
\hline $\begin{array}{l}\hat{A g e} \\
\text { (15 à } 29 \text { ans) } \\
30-39 \text { ans } \\
40-49 \text { ans } \\
50-59 \text { ans } \\
60 \text { ans et }+\end{array}$ & $\begin{array}{l}0,28 * * * \\
0,08 * * * \\
0,10 * * * \\
0,14 * * * \\
\end{array}$ & $\begin{array}{l}0,16 * * * \\
0,04 * * * \\
0,05 * * * \\
0,08 * * *\end{array}$ & $\begin{array}{l}0,36 * * * \\
0,13 * * * \\
0,07 * * * \\
0,11 * * *\end{array}$ & $\begin{array}{l}0,23 * * * \\
0,08 * * * \\
0,04 * * * \\
0,07 * * * \\
\end{array}$ & $\begin{array}{l}0,27 * * * \\
0,07 * * * \\
0,03 * * * \\
0,05 * * * \\
\end{array}$ \\
\hline $\begin{array}{l}\text { Niveau d'instruction } \\
\text { (Sans instruction) } \\
\text { Primaire } \\
\text { Secondaire et }+ \\
\end{array}$ & $\begin{array}{l}0,63 * * \\
0,80\end{array}$ & $\begin{array}{l}0,67 * * \\
0,95\end{array}$ & $\begin{array}{l}0,64 * \\
1,00 \\
\end{array}$ & $\begin{array}{l}0,64^{*} \\
1,25\end{array}$ & \begin{tabular}{|l}
0,90 \\
1,33 \\
\end{tabular} \\
\hline $\begin{array}{l}\text { Statut matrimonial } \\
\text { (Jamais marié(e)) } \\
\text { Actuellement } \\
\text { marié(e) } \\
\text { Divorcé(e), séparé(e) } \\
\text { ou veuf(ve) }\end{array}$ & $\begin{array}{l}0,11 * * * \\
0,42 * *\end{array}$ & & $\begin{array}{l}0,27 * * * \\
0,38 * *\end{array}$ & & \\
\hline
\end{tabular}

Sources : EDS du Burkina Faso 1993, 1998/1999 et 2003 (Burkina Faso 1994, 2000 et 2004).

Note : Seuils de signification: $* 10 \%(\mathrm{z}: \pm 1,645), * * 5 \%(\mathrm{z}: \pm 1,96), * * * 1 \%(\mathrm{z}: \pm 2,58)$. 
African Population Studies Vol. $22 n^{\circ}$ /Etude de la Population Africaine Vol. $23 \mathrm{~N}^{*} 2$

\section{Discussion}

L'importance des ménages de petite taille s'accroît entre 1993 et 2003, surtout en milieu urbain. Dans la capitale, la taille moyenne est passée de 5,9 à 5.5, et la proportion de ménages de moins de cinq personnes augmente de $42 \%$ (1993) à 46,8\% (2003). Alors que l'importance relative des ménages nucléaires a légèrement augmenté à Ouagadougou de 1993 (40,5\%) à 1998/1999 (41,9\%) puis à $2003(43,3 \%)$, les ménages élargis demeurent prédominants.

Les résultats des analyses par rapport à l'âge confirment ce qui était anticipé en fonction du cycle de vie des chefs de ménage : plus ils sont âgés, moins ils sont susceptibles de diriger un ménage nucléaire et de faible taille. Le statut de chef de ménage se féminise quelque peu entre $1993(11,7 \%)$ et $2003(16,9 \%)$, parallèlement à la complexification et l'agrandissement des ménages quand le chef est une femme. Cependant, les ménages dirigés par des hommes ont plus de chance d'être de grande taille, quoique les écarts entre les sexes se réduisent au fil du temps. Les hommes ont près de deux fois plus de chance d'être à la tête de ménages nucléaires, tendance qui s'accentue dans le temps. La taille moyenne des ménages dont le chef est une femme augmente de 1993 à 2003 (4,8 à 5,2 personnes), contrairement à celle des hommes $(6,0$ à $5,6)$. La complexité des ménages des femmes s'accentue; ils comportent plus souvent des petits-enfants, d'autres parents, des membres de la belle-famille et même des non parents.
Les structures familiales burkinabé ont évolué dans un contexte de crise économique associée à l'appauvrissement des ménages les mieux nantis (dont la proportion est quasi-nulle en 2003), à la diminution des ménages pauvres entre $1993(43,6 \%)$ en $2003(40,5 \%)$, et à l'augmentation de l'importance relative des ménages intermédiaires $(49,4 \%$ à $59,5 \%)$. Les ménages de faible taille (inférieure à 5) sont moins fréquents chez les nantis $(28,2 \%)$ que chez les pauvres $(44 \%)$ ou les intermédiaires $(41,8 \%)$ en 1993. C'est pourtant chez les nantis que l'augmentation des ménages de petite taille est la plus forte en 1998/1999 (36,8\% comparativement à $47,4 \%$ pour les pauvres et $46,5 \%$ pour les intermédiaires). Cette diminution générale pourrait révéler l'effritement des solidarités. L'analyse de régression démontre quant à elle que les ménages nantis ont $44 \%$ moins de chance d'être de taille réduite que les pauvres en 1993 et $41 \%$ en 1998/1999, indiquant malgré tout le maintien d'une forme de solidarité. Les ménages nantis sont dotés d'une plus grande complexité de structure, mais de moins en moins prononcée, alors qu'ils ont $41 \%$ ( $43 \%$ selon les modèles) moins de chance que les pauvres d'être nucléaires en 1998/1999, comparativement à $44 \%$ et $46 \%$ en 1993 . Par contre, plus complexes, les ménages de niveau intermédiaire ont deux fois plus de chance d'être nucléaires que les pauvres en 2003. Comme l'a indiqué la revue de la littérature, en période de crise les manifestations de solidarité des ménages aisés les amènent à être plus grands et plus complexes que les pauvres. 
Banza Baya, Danièle Laliberté: Crises des solidarités ou solidarités dans la crise ? Une lecture à partir de l'analyse de l'évolution de la structure des ménages entre 1993 et 2003 à Ouagadougou, Burkina Faso.

Si la convergence vers un système familial nucléaire était en cours au Burkina Faso, la thèse de nucléarisation voudrait aussi alors que la taille et la complexité des ménages de la capitale soient moindres dans les segments les plus éduqués de la population. Effectivement, la proportion de ménages de moins de cinq personnes domine pour les chefs ayant fait des études secondaires ou supérieures, en 1998/1999 $(56,4 \%)$ et $2003(55,4 \%)$, mais pas en 1993 $(46,7 \%)$. On constate l'inverse pour les chefs sans instruction, dont la proportion des ménages de petite taille ne dépasse jamais 40,4\%, ainsi que pour ceux de niveau primaire dont la taille se situe majoritairement entre cinq et neuf personnes $(48,8 \%$ en 1993 et $45,1 \%$ en 2003). Cependant, la structure des ménages des chefs les mieux scolarisés est généralement plus complexe; ils sont en effet plus rarement de type nucléaire. L'analyse de régression démontre une influence différentielle des niveaux de scolarité à travers le temps. Ainsi, en 1993, une instruction de niveau primaire diminue par $26 \%$ (ou $24 \%$ dépendamment des modèles) les chances de résider dans un ménage nucléaire par rapport à la catégorie sans scolarité, comparativement à 33\% en 1998/1999. Le niveau secondaire et plus est associé à un rapport de chance d'environ 0,50 (ou 0,52 dans le deuxième modèle) en 1993, mais à une valeur oscillant de 0,42 à 0,45 en 19984999 et de 0,47 en 2003. En 1993, les chefs de ménage de niveau primaire ont $37 \%$ (ou $33 \%$ selon le modèle) moins de chance que les chefs sans instruction de vivre dans un ménage de petite taille, et $36 \%$ en 19984999.

\section{Conclusion}

Notre étude avait pour objectif d'analyser l'évolution de la structure et de la taille des ménages à Ouagadougou de 1993 à 2003, à la lumière du paradigme de la nucléarisation de la famille. Il ressort que la majorité des ménages sont non nucléaires, même si les ménages nucléaires ont légèrement augmenté. Parallèlement, la taille moyenne des ménages a très peu varié au cours de la décennie. Les ménages de grande taille (cinq personnes et plus) demeurent majoritaires, avec une légère augmentation des ménages de petite taille (moins de cinq personnes). Les résultats de l'analyse multivariée démontrent que les ménages nantis ont moins de chance d'être nucléaires et de petite taille, que ceux dont le chef a une instruction plus importante (secondaire et plus) ont aussi moins de chance d'être nucléaires. Les ménages de niveau intermédiaire ont presque deux fois plus de chance d'être nucléaires que les pauvres en 2003. La majorité des ménages reste dirigée par des hommes, quoique l'importance relative des femmes chefs augmente. La proportion des femmes chefs n'a pas atteint le $20 \%$ qu'elle représenterait en Afrique subsaharienne selon Pilon et al. (1997). Â la lumière de notre étude, il serait peu osé de prétendre à une nucléarisation des ménages à Ouagadougou. D'autres recherches sur les structures familiales devraient être renouvelées dans le contexte burkinabé et ailleurs, et, si possible, à partir de sources mieux adaptées que les EDS. Leur pertinence est d'autant plus vraie qu'elles faciliteraient l'adaptation des politiques économiques et familiales en fonction de la dynamique des structures 
African Population Studies Vol. $22 n^{\circ} 2 /$ Etude de la Population Africaine Vol. $23 N^{* 2}$

familiales dans des contextes de crise économique.

\section{Références bibliographiques}

Adjamagbo-Johnson, B. 1997, "Législations et changements familiaux en Afrique subsaharienne francophone" pp. 239256. In Ménages et familles en Afrique: approches des dynamique contemporaines. Séminaire CEPEDENSEA-INS-ORSTOM-URD. Lomé 4-8 décembre 1995. Compilés par M. Pilon, T. Locoh, É. Vignikin et P. Vimard. CEPED. Les Études du CEPED. No. 15.

Baya, B. 2003, Crise des solidarités ou solidarités dans la crise? Une lecture à partir de l'analyse de la structure des ménages à Bobo-Dioulasso. AUF. Cinquièmes Journées scientifiques du Réseau de Démographie, Familles au Nord, Familles au Sud. Marseille. France 23-26 juin 2003.

Bongaarts, J. 2001, "Household size and composition in the developing world in the 1990s" Dirasat Sukkaniyah 55, (3): 263-279.

Burkina Faso 2004, Enquête démographique et de Santé 2003. Institut National de la Statistique et de la Démographie. Ministère de l'Économie et du Développement, Ouagadougou et Macro International Inc.: Calverton.

Burkina Faso 2003a, Cadre stratégique de lutte contre la pauvreté (révision 2003). Ministère de l'Économie et $\mathrm{d} u$ D é ve lo p p e m e $\mathrm{n} t$, Ouagadougou.

Burkina Faso 2003b, La pauvreté en 2003. INSD.

Burkina Faso 2000, Enquête démographique et de Santé 1998-99, Institut National de la Statistique et de la Démographie. Ministère de l'Économie et des Finances, Ouagadougou et Macro International Inc.: Calverton.

Burkina Faso 1994, Enquête démographique et de Santé 1993. Institut National de la Statistique et de la Démographie, Ministère de l'Économie et des Finances, Ouagadougou et Macro International Inc.: Calverton.

Caldwell, J.C. 1978, “A Theory of Fertility: From High Plateau to Destabilization" Population and Development Review, 4 (4):553-577.

Caldwell, J.C. 1976, “Toward a Restatement of Demographic Transition Theory" Population and Development Review, 2 (2-3): 321366.

Cordell D. and Piché V., 1997, "Pour une histoire de la famille en Afrique" pp. 55-74. In Ménages et familles en Afrique: approches des dynamiques contemporaines. Séminaire CEPEDENSEA-INS-ORSTOM-URD. Lomé 4-8 décembre 1995. Édité par 
Banza Baya, Danièle Laliberté: Crises des solidarités ou solidarités dans la crise ? Une lecture à partir de l'analyse de l'évolution de la structure des ménages entre 1993 et 2003 à Ouagadougou, Burkina Faso.

M. Pilon, M., T. Locoh, É. Vignikin et P. Vimard. CEPED. Les Études du CEPED. Numéro 15.

Debpuur, C.Y. 1998, Household Structure, Women's Autonomy and, Child Health in Ghana, DAI-A. 58(07). PhD Degree, Brown University.

Develay, A., Sauerborn, R., Diesfield, H.J. 1996, "Utilization of health care in an African urban area: results from a household survey in Ouagadougou, Burkina Faso" Social Science \& Medicine. England 43(11): 1611-1619.

Kocher, J.E. 1973, "Rural Development, Income Distribution, and Fertility Decline" New York. Population Council.

Locoh, T. 1988, "L'analyse comparative de la taille et de la structure des ménages" pp. 5.2.117-142. In Congrès africain de population, 7-12 novembre 1988. Volume II, UIESP. Dakar.

Nations unies 1993, Annuaire démographique, Nations unies, Division de la statistique. New York.

Parsons, T. and Bales, R. 1955, Family,
Socialization and Interaction Process.

Peek, C.W., Koropeckyj-Cox, T., Zsembik, B.A and Coward, R.T. 2004, "Race Comparisons of the Household Dynamics of Older Adults" Research on Aging 26 (2) March: 179201.

Pilon, M. and Vignikin, K. 2006, Ménages \& Familles en Afrique subsaharienne, Savoirs francophones, Démographie \& Développement. Éditions des archives contemporaines et AUF.

Poirier, J. 1986, “Éléments pour une problématique matérialiste de la reproduction humaine" pp. 273299. In D. Gauvreau, J. Gregory, M. Kempeneers and V. Piché (eds), Démographie et sous-développement dans le Tiers-Monde. Center for Developing Area Studies.

Roy, L. 1985, Quelques aspects du cycle de vie des ménages au Burkina Faso. Mémoire présenté à la Faculté des études supérieures en vue de l'obtention du grade de M.Sc.. Université de Montréal. 